Mots. Les langages du politique

$94 \mid 2010$

Trente ans d'étude des langages du politique

(1980-2010)

\title{
Vie et mort du citoyen cathodique
}

Jean-Claude Soulages

\section{OpenEdition}

Journals

Édition électronique

URL : https://journals.openedition.org/mots/19874

DOI : $10.4000 /$ mots. 19874

ISSN : 1960-6001

Éditeur

ENS Éditions

\section{Édition imprimée}

Date de publication : 30 novembre 2010

Pagination : 125-132

ISBN : 978-2-84788-235-3

ISSN : 0243-6450

Référence électronique

Jean-Claude Soulages, "Vie et mort du citoyen cathodique », Mots. Les langages du politique [En ligne],

94 | 2010, mis en ligne le 06 novembre 2012, consulté le 29 avril 2022. URL : http://

journals.openedition.org/mots/19874; DOI : https://doi.org/10.4000/mots.19874 


\section{Mots \\ Les langages du politique}

№ 94 novembre 2010

\section{Trente ans d'étude des langages du politique (1980-2010)}

ouvrage coordonné par Paul BACOT, Marlène Coulomb-GulLY, Jean-Paul Honoré, Christian Le BART, Claire Oger, Christian Plantin

\section{SOMMAIRE}

Paul Bacot, Marlène Coulomb-Gully, Jean-Paul Honoré, Christian Le Bart, Claire Oger, Christian Plantin

Le discours politique n'est pas transparent. Permanence

et transformations d'un objet de recherche

OUTILS ET ENJEUX DU DISCOURS POLITIQUE

Ruth Amossy, Roselyne Koren

Argumentation et discours politique

Christian Plantin

Argumentation-rhétorique. Les eaux mêlées

Caroline Ollivier-Yaniv

Discours politiques, propagande, communication, manipulation 
Marc Bonhomme

La caricature politique

Paul Bacot

Développement et diversification d'une onomastique politique

Ruth Wodak

The Discursive Construction of History. Brief Considerations

Henri Boyer

Les politiques linguistiques

\section{LIEUX DE LA PRODUCTION DU DISCOURS POLITIQUE}

Christian Le Bart

Parler en politique

Dominique Maingueneau

Le discours politique et son «environnement»

Alice Krieg-Planque, Claire Oger

Discours institutionnels. Perspectives pour les sciences

de la communication

Sophie Béroud, Josette Lefèvre

Le corpus syndical. Une expérience au long cours

Corinne Gobin, Jean-Claude Deroubaix

L'analyse du discours des organisations internationales.

Un vaste champ encore peu exploré

François de la Bretèque

Le retour de la parole politique dans le cinéma français

Jean-Claude Soulages

Vie et mort du citoyen cathodique

Jacques Guilhaumou

Les discours de la Révolution française.

Aperçu d'ensemble d'un trajet de recherche (1980-2009) 


\section{DISCIPLINES ET CHAMPS DE RECHERCHE POUR L'ÉTUDE DES LANGAGES DU POLITIQUE}

Philippe Braud

L'apport de la science politique à l'étude des langages du politique

Claire Blandin

L'apport de l'histoire des médias à l'étude des langages du politique

Jean-François Tétu, Bernard Lamizet

Les SIC et les langages du politique

Sylvianne Rémi-Giraud

Sémantique lexicale et langages du politique.

Le paradoxe d'un mariage difficile?

Marlène Coulomb-Gully, Juliette Rennes

Genre, politique et analyse du discours.

Une tradition épistémologique française gender blind

Johannes Angermüller

Analyser le discours politique en Allemagne (1980-2010)

Érik Neveu

L'apport de Pierre Bourdieu à l'analyse du discours.

D'un cadre théorique à des recherches empiriques

Roselyne Ringoot

Questionner le discours avec Michel Foucault.

Actualisations théoriques et actualité éditoriale

\section{ENTRETIEN}

Maurice Tournier

Mots et politique, avant et autour de 1980

La revue Mots. Les langages du politique encourage l'usage des rectifications de l'orthographe proposées par le Conseil supérieur de la langue française et approuvées par l’Académie (Journal officiel, $n^{\circ}$ 100, 6 décembre 1990). 


\section{Vie et mort du citoyen cathodique}

La rencontre de l'analyse de discours et des études télévisuelles a confronté très tôt les chercheurs à une série de recadrages tant d'ordre méthodologique que théorique. Historiquement née et élaborée à partir de travaux portant sur des matériaux langagiers issus de l'écrit ou bien de l'oral, l'analyse de discours, en abordant l'objet télévisuel, court sans cesse le risque de succomber à deux biais découlant de deux présupposés : un présupposé d'ordre épistémologique - la signification discursive s'imposerait à travers les filtres de la langue, voire de la parole - et un présupposé d'ordre méthodologique : cette seule signification langagière transcenderait la dimension médiatique des productions discursives observées. Avec, pour conséquence, la réduction de ces productions soit à des textes clos, soit à des postures énonciatives hégémoniques, ou bien encore à des effets d'influence supposés réalisés. De ce recadrage théorique découlent plusieurs séries d’implications.

Tout d'abord, il faut rappeler que toute opération de mise en images relève plus d'un procédé de médiation et de mise en discours que d'une visée prescriptive de référentialité. La vision humaine s'accompagne toujours d'un procédé inférentiel de mise en situation et n'est en aucun cas la résultante de la somme d'images d'une quelconque réalité. Elle correspond avant tout à un processus particularisant d'extraction d'informations basée sur un système perceptuel sophistiqué porté par un corps constamment inséré au cœur d'un environnement (Gibson, 1989). Regarder la télévision n'est donc jamais réductible à un phénomène purement cumulatif d'arrêts sur image et se réduit plutôt à une opération dynamique de discrimination de formes, de surfaces, d'objets, de textures mais surtout de vecteurs d'orientation reposant sur une interaction avec un corps. Le téléspectateury est désigné et contractuellement encadré par des instructions de sens d'ordre iconico-cinétiques qui concourent à générer une visée de coprésence à l'intérieur des émissions (adresses verbales, regard adressé, posture frontale, asservissement des mouvements du cadre au point de vue frontal du téléspectateur, etc.). Le flux des programmes porte l'empreinte de cette topologie imaginaire de «contact indiciel» (Véron, 1984) qui nourrit la performativité de «cadres participatifs» (Livingstone, Lunt, 1994) des plus 
disparates (celui du journal télévisé, des émissions de variétés, des débats, etc.). Cette association empathique entre ce cadre instrumentalisé et son contrechamp situé hors cadre qu'est le regard du téléspectateur est décisive. Ainsi, tour à tour, le petit écran peut se métamorphoser en fenêtre, en tribune, en véhicule, en loupe, en support scriptural pour son destinataire (Soulages, 2007). II convient donc prioritairement de prendre en compte ces instructions de sens propres au support médiatique et non plus, par un effet de mimétisme, de s’évertuer à recourir à un décalque des structures de la langue ou bien à des instruments d'analyse empruntés au genre audiovisuel dominant (le cinéma narratif).

En deuxième lieu, la plupart des images télévisuelles ne peuvent en aucun cas être considérées comme le seraient des icônes isolées. Du fait qu'elles sont doublement dépendantes. Tout d'abord, d'un réseau de diffusion-réception et de stratégies de programmation sans lesquels elles n'auraient pas d'existence concrète et en dehors desquels elles ne possèdent pas d'autonomie. Et surtout parce que les images télévisuelles dépendent pour une grande part des univers génériques que le média déploie à travers la dynamique de multiples «cadres participatifs» (éphémères et contextuels) sous-tendus par des «contrats de communication» (Charaudeau, 1997) dédiés à leurs communautés de public. Prosaïquement, le téléspectateur demeure toujours prisonnier d'une grille de programmes.

Ainsi, toute production télévisuelle peut être considérée comme l'accomplissement d'une performance publique programmée. Daniel Dayan (1984, p.149) a contribué à dissiper cette confusion tenace qui persiste du côté de la réception des productions médiatiques : «Les spectacles à public reposent sur une performance du spectateur comme membre d'un public, sur une performance publique d'un spectateur. Les spectacles à spectateur voient une telle performance disparaître pour être reprise en charge, simulée par le texte. Tout se passe comme si la participation de ce dernier n'était qu'une intériorisation, qu'une transposition sur le registre privé d'une performance sociale.» En un mot, tout programme de télévision repose sur une performance du téléspectateur comme membre d'une communauté de public. Le média télévisuel ne fait ainsi que pragmatiquement démultiplier le «cadre d'usage » (Flichy, 1995) hégémonique qu'avait contribué à instituer le cinéma - la fiction et son spectator in fabula hérité du genre romanesque - en proposant pour ce téléspectateur «performé » de nouvelles logiques d'usage : informative, ludique, compassionnelle, etc. Cette hétérogénéité structurelle des productions télévisuelles exemplifie au plus haut point ce «cracking analytique » de contenus et de formes qu'Edgar Morin postulait comme moteur générateur des produits de la culture de masse, basé sur «un dialogue permanent entre une production et une consommation » (Morin, 1962, p.60).

Or, sur le plan de la production et de la circulation de ressources cognitives, nos sociétés, depuis le siècle dernier, se caractérisent par l'hégémonie pro- 
gressive de ces performances des réseaux télévisuels qui sont devenus des acteurs décisifs de l'espace public. Leur quadrillage du territoire national à partir de dispositifs centralisateurs de diffusion a permis de façon quasi indolore de conquérir tous les espaces périphériques. Ce rôle d’interfaces (Véron, 1989) qu'ils assument a donné le jour à une réflexivité sociale dont la complexité est très éloignée de la rationalité argumentative que postulait l'espace public habermassien. On peut en effet opposer à cette notion abstraite et inerte celle d'une sphère publique conçue comme « une arène publique tout à la fois conflictuelle, asymétrique, plurielle, stratégique et plastique» (Macé, 2006, p.32). Cette médiatisation progressive des sociétés modernes a permis de quitter l'ère des masses sédentaires pour celle des audiences, des publics, désormais construits comme des collectivités hétérogènes et réflexives de citoyens. Les médias électroniques, la radio et surtout la télévision, ont contribué à nourrir ce processus de déterritorialisation et de médiation en abolissant la pesanteur des distances et en court-circuitant l'espace académique des institutions représentatives. Le suffrage universel avait accouché du citoyen politique, avatar juridique et national, les médias de masse ont accouché d'un citoyen cathodique mis en réseau, conscience standardisée et perfusée par les récits d'une même actualité. En rendant visible le pouvoir et ses protocoles, ces interfaces médiatiques ont fait exister subsidiairement la démocratie d'opinion et du même coup porté à l'existence de nouveaux acteurs, les experts, les sondeurs, les conseillers, tous porte-parole de «conflits de définition» du social (Beck, 2001) qui se sont mis à apparaître en pleine lumière. Au centre de cette opération de médiation, à maintes occasions, les journalistes de télévision sont devenus à leur corps défendant les ventriloques de ces instances.

Car au-delà du simple téléspectateur membre d'une audience, la plupart des programmes impliquent avant tout l'acteur social et culturel, membre de l'espace public d'une société avec toutes les visées d'influence qui traversent ce dernier et dont la télévision, pour le discours politique, demeure encore aujourd'hui la pièce maîtresse. Comme le constate Jésus Martin Barbero, ces dispositifs de médiation ont poussé à «la transformation de l'idée politique de nation en vécu, sentiment et quotidienneté » (Barbero, 2002, p. 155). Tout à fait ambivalents face au média télévisuel, les élus de la République ont toujours annexé cet espace public médiatique en en faisant le pré carré de l’État français ${ }^{1}$. Bon an mal an, tout en articulant la sphère publique à celle de la consommation quotidienne de spectacles, la télévision a été un agent actif de la médiatisation du politique. Enfermés dans leur cadre-fenêtre,

1. La France est, parmi les pays démocratiques, le seul où le studio du Vingt heures soit grand ouvert aux prestations des politiques. La forme d'adresse au public qu'avait inauguré Alain Peyrefitte, ministre de l'Information en 1965, semble toujours rémanente. En effet, des ÉtatsUnis à l'Espagne, ce territoire est exclusivement réservé aux journalistes (les acteurs politiques tiennent des conférences de presse devant toute la presse). 
les acteurs politiques ont, à tour de rôle, imposé chacun leur rituel d'accès aux citoyens cathodiques, faisant alterner la cérémonie, la proximité ou bien l'empathie. En retour, l'acteur politique a été contraint d'abandonner les coulisses des palais de la République pour faire irruption sur cette scène de la sphère du quotidien et du divertissement et surtout, celui-ci a été incité à planifier et quantifier la productivité de ses rencontres épisodiques avec les citoyens cathodiques.

Or, ironiquement, le média télévisuel, principal acteur de cette nouvelle gestion de l'image publique de la politique et de son incarnation, est devenu aujourd'hui la première victime de sa créature, car cette dernière, qu'il a laborieusement contribué à façonner, est désormais libre de choisir et de faire son marché, toute seule. Délaissant le canal centralisateur originel qui lui a donné le jour, le citoyen cathodique peut désormais exercer ses choix sur d'autres écrans. On perçoit bien alors tout ce qu'il peut y avoir de consubstantiel entre ce lent processus d'individuation et d'éducation du télé-citoyen et le fonctionnement de nouvelles interfaces qui se proposent non plus d'enchaîner ou de réunir les publics, mais de mettre en réseaux des individualités en multipliant les liens sociaux. Le citoyen cathodique peut désormais s'affranchir de son mentor et donc passer à l'acte en devenant télécide.

\section{La mort programmée du journal télévisé}

On peut observer la trace de cette déliaison progressive à travers l'évolution des programmes télévisés, de leurs enjeux mais aussi de leurs usages. C'est le cas des émissions politiques qui ont longtemps escorté les programmes de la télévision publique, étroitement encadrées et, aux yeux de certains, implicitement instrumentalisées par les pouvoirs politiques. Or paradoxalement, si ces performances ont pu jouer un rôle décisif dans la publicisation du discours politique, elles ont surtout œuvré à l'apprivoisement du débat public. Marginalisées par la course à l'audience consécutive à la privatisation des réseaux, elles ont laissé le champ libre à d'autres dispositifs et modes d'expression plus spectaculaires et attractifs de la discursivité sociale, caractérisés par l'intrusion de la parole profane et de l'expérience privée (Lochard, Soulages, 2003). Paroles épiphaniques d'individus-citoyens révélant «l'immédiateté de leur expérience vécue, seule vérité, contre la citoyenneté définie comme transcendance et abstraction» (Schnapper, 2002, p. 252).

A contrario, certains programmes affichent toujours cette conformité par rapport à leur matrice originelle et n'ont par contre pratiquement pas subi d'évolution. À commencer par le plus important d'entre eux en termes d'audience, le journal télévisé national. En effet, ce dernier reste redevable de la rémanence d'un «transgenre» (Steimberg, 1997), typique de la modernité, le 
journal national d'information. Successivement, imprimé, radiodiffusé, filmé et télévisé, ce genre médiatique a été historiquement structuré et adossé à la constitution, typique du creuset idéologique des États-Nations, d'une même «communauté imaginée» (Anderson, 2002). À force de répéter aux téléspectateurs qu'ils existaient et comptaient - «les Français pensent que... »-, Yves Mourousi, Roger Gicquel et bien d'autres ont inventé ce «Français moyen », assimilé à la voix de l'opinion, disséminé dans la nébuleuse des microtrottoirs, des sondages et des assertions d'experts. Le citoyen cathodique venait de voir le jour. Paradoxalement, les politiques ont ignoré cette activité performative du média et n'ont perçu dans ce processus que la «voix de la France», celle de l'État-Nation dont Georges Pompidou affublait l'ORTF. Or, cette voix est restée toujours quasi aveugle, une voix sans images, faisant fi de la silhouette et des visages de son public. Tout acte performatif, comme l'avance Judith Butler, «n'est pas simplement une pratique rituelle: c'est l'un des rituels majeurs par lesquels les sujets sont formés et reformulés» (Butler, 2004, p. 247). Bâti sur le moule des États-Nations - un émetteur puissant et des récepteurs faibles -, ce schéma caractéristique du média télévisuel apparaît aujourd'hui suranné.

Certes, nombreux avaient été ceux qui avaient anticipé cet état de fait, en pointant les failles du Vingt heures. Le carrefour fédérateur du prime time laisse non seulement peu de temps au développement analytique et fouillé des nouvelles, mais réduit la couverture du spectre événementiel à une poignée de sujets attendus et nationalement consensuels.

Sa seconde faiblesse découle de son fonctionnement structurel, le journal télévisé est resté pour l'essentiel une plate-forme de redistribution de flux d'information, à son corps défendant, du fait de son inertie et surtout de sa visibilité encombrante. La rédaction d'un Vingt heures ne peut que rarement produire du scoop ou bien de l'enquête; elle excelle par contre dans ce que l'on peut appeler des événements programmés : conférence de presse, inaugurations, événements publics, etc. Ce biais médiologique s'avère tout à fait contre-productif car il entre immédiatement en résonance avec l'agenda des acteurs sociaux et souvent avec celui des acteurs politiques de l'État-Nation (Leblanc, 1987). Si l'on en dresse une comptabilité au jour le jour, l'actualité télévisée est le plus souvent une actualité nationale systématiquement programmée, quasi prédictible².

Mais surtout, d'autres pratiques et de nouveaux concurrents sont apparus. Déjà, les chaînes d'information continue avaient contribué à faire voler en éclats le modèle du rendez-vous immuable de la Nation. En opérant un holdup permanent sur l'actualité et souvent sur son commentaire, elles ont durablement démonétisé la grande messe du Vingt heures et contribué à susciter

2. Pour preuve, la plupart du temps, les conducteurs des trois principaux journaux télévisés nationaux sont quasi identiques et leur ligne éditoriale équivalente. 
des pratiques et des publics de plus en plus nomades. Certaines d'entre elles (CNN, Euronews, El Jazira, voire ARTE) ont de surcroît contribué à fortement provincialiser le journal national franco-français.

Enfin, la concurrence d'Internet travaille à moyen terme à fragiliser durablement ce modèle. Le recueil d'information n'y connaît pas ces « conditions a priori » qu'imposent les logiques et l'agenda national du média télévisuel. La Toile peut tout aussi bien colporter des images volées, des informations brutes, des reportages, des analyses, voire des encyclopédies. Ce point de vue transversal parfois direct et quasi synoptique qu'offre la navigation de site en site est tout à fait aux antipodes du modèle institutionnel que délivre le journal télévisé national, toujours pyramidal. À l'agenda de l'État-Nation, le web tend à substituer l'agenda de la nébuleuse aléatoire et pluriphonique de sources indépendantes et périphériques, mais aussi de réseaux sociaux.

\section{Hybridité et transversalité}

Tous ces éléments ont pu concourir à fragiliser le format du journal télévisé - parangon de la modernité et vitrine des sociétés développées depuis les années soixante. Mais, simultanément, ce qui a été décisif tient à ce qui s'est joué pour les publics, tant du côté des ressources d'interaction qui leur sont offertes par les nouvelles interfaces médiatiques que des identités émergentes qui se révèlent au grand jour dans les nouveaux programmes de collecte de l'expression sociale. La conception positiviste et monolithique de la «communauté imaginée » de la modernité nationale dont ont accouché les années soixante n’a pas résisté aux bouleversements de la fin du siècle, la globalisation, la multiplication des technologies nomades; tout le paysage médiologique et culturel s'en est trouvé transformé, accompagnant l'éclatement des identités sociales, sexuelles, culturelles. L'individu a cessé d'être conçu seulement comme un sujet politique abstrait : il est désormais une personne privée, incarnée, charnelle, assujettie non plus à l'agenda de l'universel mais aux répertoires individuels d'identités plurielles faites de désirs et de besoins particularisants. Comme le constate Axel Honneth «ces percées individuelles visant à élargir les rapports de reconnaissance se rassemblent en un nouveau système d'exigences normatives, et la succession de ces systèmes pousse constamment l'ensemble du développement social dans le sens d'une individualisation croissante » (Honneth, 2008, p. 102). Manifestement, ce processus entre en opposition avec la machine à produire un consensus national univoque qu'a représenté le journal télévisé dès ses origines. Face à une démocratie médiatique réticulaire et à ces reconfigurations multiples des identités individuelles, son agenda centralisateur et monolocutif s'avère incapable d'intégrer de telles transformations car elles sont avant tout locales, hétérogènes, minoritaires et souvent dissensuelles. Aux détriments du modèle 
centralisateur et homogénéisant du "panoptique inversé» (Allemand, 1980), ceux du réseau et du marché semblent l'avoir définitivement emporté. Car cette transformation du paysage médiatique et culturel, tout comme l'éclatement des identités sociales, sexuelles, culturelles, entrent en opposition avec la machine à produire un consensus national univoque qu'a représenté le journal télévisé dès ses origines. Ce que l'on pourrait appeler, en reprenant les termes de Michel Callon, des «forums hybrides» (Callon, 2001) - nouveaux lieux non institutionnels de circulation d'information et de débat, symptomatiques du rejet des médiations institutionnelles, celle du politique ou bien du savoir académique -, sont bien à mille lieux du format académique du journal télévisé national.

La mise en place d'un réseau de médias mondial contribue chaque jour davantage à détacher les parcours individuels des publics de leur territoire national, comme la télévision l'avait déjà accompli pour les appartenances locales. Tandis que le politique agit (encore en partie) dans le cadre de l'État-Nation, le monde vécu et l'environnement des individus, tant dans leurs ressources matérielles qu'imaginaires, est déjà grand ouvert à une société réticulaire et globale. Cette déterritorialisation de l'environnement du citoyen a pour effet immédiat que le territoire imaginaire qu'ont laborieusement institué les ÉtatsNations est aujourd'hui de plus en plus poreux. Les mailles du filet à travers lesquelles les individualités sont mises en réseaux ainsi que les frontières des nouveaux territoires et des nouvelles identités sont de plus en plus lâches et hybrides'. À tel point qu'aujourd'hui, bien souvent, la périphérie semble l'avoir définitivement emporté sur le centre. Si la sphère publique de nos sociétés s'affiche bel et bien comme un espace de libre circulation de discours, elle constitue tout autant un révélateur efficace quant aux opérations de médiation qui s'y déploient et à travers lesquelles les interfaces médiatiques distribuent et assignent des places à leurs audiences, mais aussi aux citoyens.

\section{Références}

Allemand E., 1980, Pouvoir et télévision. Les machines d'organisation, Paris, ÉtatsNations.

ANDERSON B., 2002, L'imaginaire national. Réflexions sur l'origine et l'essor du nationalisme, Paris, La Découverte.

BArbero J. M., 2002, Des médias aux médiations. Communication, culture et hégémonie, Paris, CNRS.

3. La performance politique télévisuelle du président de la République le 25 janvier 2010, qui a réuni 8,6 millions de téléspectateurs, alliait le dispositif du journal télévisé à celui de l'émission de spectacle (Paroles de Français), avec en amont l'apport du réseau social Facebook et en aval l'incarnation cathodique d'un panel de Français moyens. 
BECK U., 2001, La société du risque. Sur la voie d'une autre modernité, Paris, Alto/ Aubier.

CAllon M., 2001, Agir dans un monde incertain. Essai sur la démocratie technique, P. Lascoumes, Y. Barthe éd., Paris, Le Seuil.

BUtLER J., 2004, Le pouvoir des mots. Politique du performatif, Paris, Éditions Amsterdam.

ChARAUdeau P., 1997, Le discours d'information médiatique. La construction du miroir social, Paris, INA-Nathan.

DAYAN D., 1984, «Le spectateur performé », Hors Cadre, nº 2, p. 137-149.

Flichy P., 1995, L'innovation technique, Paris, La Découverte.

GiBson J. T., 1989, The Ecological Approach of Visual Perception, Londres, Laurence Erlbaum.

Honneth A., 2008, La lutte pour la reconnaissance, Paris, Cerf.

Leblanc G., 1987, Treize heures/Vingt heures. Le monde en suspens, Marburg, Hitzeroth.

LIVINGSTONE S., LUNT P., 1994, «Se faire entendre dans l'espace public. Les femmes, la télévision et le citoyen-téléspectateur », Réseaux, n63, p. 59-75.

LoChARd G., SOulages J.-C., 2003, "La parole politique à la télévision. Du logos à l'ethos", Réseaux, n¹08, p. 65-95.

MACÉ E., 2006, Les imaginaires médiatiques. Une sociologie postcritique des médias, Paris, Éditions Amsterdam.

MORIN E., 1962, L'esprit du temps, vol. I, Névrose, Paris, Grasset.

SCHNAPPER D., 2002, La démocratie providentielle. Essai sur l'égalité contemporaine, Paris, Gallimard.

Soulages J.-C., 2007, Les rhétoriques télévisuelles ou le formatage du regard, Bruxelles, De Boeck-INA.

Steimberg O., 1997, «Des genres populaires à la télévision. Étude d’une transposition », Réseaux, $\mathrm{n}^{\circ} 81, \mathrm{p}$. 47-61.

VÉRON E., 1984, "Le séjour et ses doubles. Architectures du petit écran », Temps libre, $\mathrm{n}^{0} 11, \mathrm{p} 67-78$.

- 1989, "Interfaces. Sur la démocratie audiovisuelle avancée », Hermès, nº 4 , Le nouvel espace public, p.113-126. 